\title{
Risco de depressão e ansiedade em gestantes na atenção primária
}

RESUMO | Objetivo: Identificar os riscos para depressão e ansiedade em gestantes de uma unidade de saúde da Atenção Primária. Métodos: Estudo descritivo exploratório de abordagem quantitativa realizado entre fevereiro a abril de 2020 em uma unidade de Saúde de Olinda-PE (Brasil); foi utilizado o instrumento validado "Cartão de Babel". A análise dos dados foi realizada pelo Statistical Package for Social Sciencies por meio do teste Qui-quadrado com correção de Yates (significância de 5\% - $p \leq 0,05$ ). Resultados: 71 gestantes foram analisadas, dentre os quais 32,3\% referiram já ter sofrido violência psicológica. Por meio do Cartão de Babel verificou-se que $49,3 \%$ das gestantes tinham alto risco para o desenvolvimento de transtorno de ansiedade (p-valor: 0,004) e 29,5\% apresentou risco moderado para depressão (p-valor: 0,004). Conclusão: Iniciativas preventivas à ocorrência da depressão e ansiedade na gestação, como o monitoramento da saúde mental e o seu rastreio durante a consulta de pré-natal são necessários.

Palavras-chaves: Gravidez; Saúde mental; Cuidado pré-natal.

ABSTRACT I Objective: To identify the risks for depression and anxiety in pregnant women in a Primary Health Care unit. Methods: Descriptive exploratory study with a quantitative approach carried out between February and April 2020 at a Health Unit in Olinda-PE (Brazil); the validated instrument "Babel Card" was used. Data analysis was performed using the Statistical Package for Social Sciencies using the Chi-square test with Yates correction (significance of $5 \%-p \leq 0.05$ ). Results: 71 pregnant women were analyzed, among which $32.3 \%$ reported having suffered psychological violence. Through the Babel Card, it was found that $49.3 \%$ of pregnant women were at high risk for the development of anxiety disorder ( $p$-value: 0.004 ) and $29.5 \%$ had a moderate risk for depression ( $p$-value: 0.004). Conclusion: Preventive initiatives for the occurrence of depression and anxiety during pregnancy, such as monitoring mental health and tracking it during the prenatal consultation are necessary.

Keywords: Pregnancy; Mental Health; Prenatal Care.

RESUMEN | Objetivo: Identificar los riesgos de depresión y ansiedad en gestantes en una unidad de Atención Primaria de Salud. Métodos: Estudio descriptivo exploratorio con abordaje cuantitativo realizado entre febrero y abril de 2020 en una Unidad de Salud de Olinda-PE (Brasil); Se utilizó el instrumento validado "Babel Card". El análisis de los datos se realizó mediante el paquete estadístico para ciencias sociales mediante la prueba de Chi-cuadrado con corrección de Yates (significancia del 5\% $\mathrm{p} \leq 0.05$ ). Resultados: Se analizaron 71 mujeres embarazadas, de las cuales el 32,3\% refirió haber sufrido violencia psicológica. A través de la Tarjeta Babel, se encontró que el $49,3 \%$ de las mujeres embarazadas tenían alto riesgo de desarrollar trastorno de ansiedad (valor p: 0,004) y el 29,5\% tenían un riesgo moderado de depresión (valor p: 0,004). Conclusión: Son necesarias iniciativas preventivas para la aparición de depresión y ansiedad durante el embarazo, como el seguimiento de la salud mental y su seguimiento durante la consulta prenatal.

Palabras claves: Embarazo; Salud mental; Atención Prenatal.

\section{Gemima Farias Pessoa da Silva}

Enfermeira graduada pela Universidade Católica de Pernambuco.

ORCID: 0000-0003-1033-3127

\section{Suzana Vitorino dos Santos}

Enfermeira graduada pela Universidade

Católica de Pernambuco.

ORCID: 0000-0003-4081-7704

\section{José William Araújo do Nascimento}

Enfermeiro (Universidade Católica de Pernambuco), Mestrando em Ciência da Computação (Universidade Federal de Pernambuco) e Membro do Grupo de Pesquisa e Estomaterapia (Universidade de Pernambuco).

ORCID: 0000-0002-1844-1117

\section{Francisca Silva de Santana}

Enfermeira da Estratégia de Saúde da Família (Olinda-PE).

ORCID: 0000-0003-2631-7295

\section{Josian Silva de Medeiros}

Fonoaudiólogo (Universidade Católica de Pernambuco), Enfermeiro (FUNESO), Mestre e Doutor em Neuropsiquiatria e Ciências do Comportamento (Universidade Federal de Pernambuco), Professor e coordenador do estágio do curso de Enfermagem da Universidade Católica de Pernambuco e Professor Titular I do Curso de Medicina da Faculdade Tiradentes (FITS). ORCID: 0000-0003-2194-4150

\section{Suzane Brust de Jesus}

Enfermeira (Unisinos), especialista em Saúde Pública (FIOCRUZ), especialista em Saúde da Família (Universidade de Pernambuco), Mestre em Linguística (Universidade Federal da Paraíba) e docente em Enfermagem e Medicina da Universidade Católica de Pernambuco. ORCID: 0000-0002-3826-8200

Recebido em: 06/09/2020

Aprovado em: 23/09/2020
INTRODUÇÃO

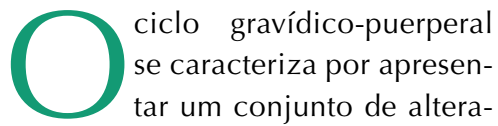
ções fisiológicas, psíquicas, hormonais e sociais que permeam um aumento no risco para o sofrimento emocional neste período de vida da mulher ${ }^{(1)}$.

Algumas mulheres recebem o período gestacional com nostalgia, satisfação pessoal e familiar, porém, este momento é de maior vulnerabilidade para o aparecimento ou recaída de um agravo psíquico, como a ansiedade e depressão ${ }^{(2,3)}$.

As evidências científicas acerca da ansiedade entre mulheres gestantes ainda são limitadas, em detrimento da de- 
pressão pré-natal, porém seus sintomas são relativamente comuns, variando de leves a graves ${ }^{(3)}$.

A depressão e a ansiedade podem estar associadas ao trabalho de parto prematuro, baixo peso ao nascer e problemas no desenvolvimento da criança. Nas gestantes, aumentam o risco de exporem-se ao tabaco, álcool e outras drogas, além do risco de desnutrição e a dificuldade de seguir orientações dadas no pré-natal de rotina, diminuindo inclusive a frequência nessas consultas, o que tem sido associado ao risco de mortalidade neonatal ${ }^{(3,4,5,6)}$.

A preocupação em avaliar a saúde mental das gestantes, infelizmente tem despertado pouca atenção entre a sociedade, isto por que as próprias mulheres podem relutar em compartilhar sintomas de tristeza e irritabilidade, além de haver uma tendência a se concentrar na saúde física (materna e fetal) durante a gravidez, e não na saúde mental ${ }^{(3)}$.

Diante disso, é fundamental que haja um rastreio para identificar o risco de depressão e ansiedade nas gestantes e que esses sinais sejam precocemente detectados, com o objetivo de encaminhar aos profissionais especializados para diagnóstico e tratamento imediato, evitando assim, consequências futuras ao binômio mãe-filho.

Ante o exposto, questionou-se: Qual os riscos para depressão e ansiedade entre gestantes acompanhadas em uma unidade de saúde de Olinda-PE? Desta forma este estudo objetivou identificar os riscos para depressão e ansiedade em gestantes de uma unidade da Atenção Primária à Saúde do referido município do Nordeste do Brasil.

\section{MÉTODOS}

Trata-se de um estudo descritivo-exploratório de abordagem quantitativa, realizado em uma Unidade de Saúde da Família (USF) do município de Olinda-PE, região metropolitana do Recife (Brasil).
Diante disso, é fundamental que haja um rastreio para identificar o risco de depressão e ansiedade nas gestantes e que esses sinais sejam precocemente detectados, com o objetivo de encaminhar aos profissionais especializados para diagnóstico e tratamento imediato, evitando assim, consequências futuras ao binômio mãe-filho.
A amostra desta pesquisa foi composta por gestantes em acompanhamento pré-natal, de modo que a população foi representada por aquelas atendidas na USF de realização do estudo no período de fevereiro a abril de 2020. Foram incluídas gestantes com idade igual ou superior a 18 anos e aquelas atendidas no pré-natal de baixo risco. Excluiu-se aquelas que já tinham diagnósticos prévios de agravos psiquiátricos e/ ou que já faziam tratamento farmacológico para transtornos mentais.

Para condução do estudo entre as gestantes foram utilizados dois instrumentos de coleta de dados:1) Formulário de variáveis sociodemográficas e da história da gesta atual (desenvuelto pelos pesquisadores); 2) Cartão Babel. Este último é um instrumento validado, de rastreio para avaliação em saúde mental na Atenção Primária, adaptado pelo Ministério da Saúde do Brasil ${ }^{(7)}$. Para este estudo, foi utilizado apenas as variáveis de triagem para depressão e ansiedade.

Todos os dados coletados foram organizados e armazenados em um banco de dados estruturado no Microsoft Excel 2016. Posteriormente, foram analisados no programa Statistical Package for Social Sciencies (SPSS), versão 23.0. Para analisar a independência entre as variáveis explanatórias foi utilizado o teste Qui-quadrado com correção de Yates. O nível de significância de 5\% foi considerado em todos os testes estatísticos e as relações foram consideradas estatisticamente significativas para $\mathrm{p} \leq 0,05$.

O referido estudo foi aprovado pelo Comitê de Ética em Pesquisa da Universidade Católica de Pernambuco, de acordo com as orientações da Resolução 466/12 do Conselho Nacional de Saú$\mathrm{de}^{(8)}$, com número do parecer 3.931.267 (CAAE: 29149220.0.0000.5206).

\section{RESULTADOS}

A população desta pesquisa foi 
Tabela 1: Triagem para o transtorno de ansiedade entre gestantes, conforme instrumento "Cartão Babel", Olinda, PE, Brasil, 2020 (N: 71).

Variáveis do instrumento sobre Ansiedade

N $\%$ p-valor*

Você tem se preocupado demais?

\begin{tabular}{lll} 
Sim & 51 & 71,8 \\
Não & 20 & 28,2 \\
\hline Você tem se sentido esgotada ou tensa? & 33 & 46,5 \\
Sim & 38 & 53,5 \\
\hline Não & & \\
Você tem se sentido muito irritada? & 42 & 59,2 \\
\hline Sim & 29 & 40,8 \\
\hline Não & & \\
\hline Você tem tido dificuldade em relaxar? & 29 & 40,8 \\
\hline Sim & 42 & 59,2
\end{tabular}

Você tem sentido tontura, suor frio, diarreia, formigamentos ou desconforto no estômago?

\begin{tabular}{lll} 
Sim & 27 & 38,0 \\
\hline Não & 44 & 62,0 \\
\hline Você está preocupado com sua saúde & & \\
\hline Sim & 31 & 43,7 \\
\hline Não & 40 & 56,3 \\
\hline $\begin{array}{l}\text { Estes problemas têm prejudicado sua qualidade de vida e rela- } \\
\text { ção com outras pessoas? }\end{array}$ & \\
\hline Sim & 13 & 18,3 \\
\hline Não & 58 & 81,7
\end{tabular}

Triagem (número de resposta positiva por gestante)

\begin{tabular}{|lll|l} 
Nenhum risco $(0-4)$ & 36 & 50,7 & 0,004 \\
Possível risco forte $(\geq 5)$ & 35 & 49,3 &
\end{tabular}

Nota: *Teste Qui-Quadrado com correção de Yates

Fonte: Elaborada pelos autores.

Tabela 2: Triagem para depressão entre gestantes, conforme instrumento "Cartão Babel", Olinda, PE, Brasil, 2020. (N: 41)

Variáveis do instrumento sobre Ansiedade

N $\%$

\section{(A) Seu apetite mudou de forma significativa?}

Sim

$25 \quad 60,9$ composta por 71 gestantes; estas eram predominantemente mulheres jovens com idade entre 20 e 30 anos $(53,5 \%)$ e solteiras (80,3\%). 46,5\%, das gestantes estavam no $2^{\circ}$ trimestre de gestação e tinham entre 2 e 3 filhos $(47,9 \%)$.

Ao ser realizado a triagem de ansiedade entre as gestantes, conforme a Tabela 1, verificou-se que $71,8 \%$ das gestantes afirmaram que "tem se preocupado demais". Mediante o Cartão Babel, 35 gestantes (49,3\%) apresentaram escore igual ou maior a 5 na pontuação, ou seja, com possível risco forte para ansiedade. Após aplicação do teste Qui-quadrado com correção de Yates, verificou-se um significativo valor estatístico sobre as variáveis encontradas (p-valor: 0,004).

Quanto à triagem de depressão, o Cartão Babel apresenta duas perguntas iniciais relacionadas ao estado de emoção da gestante. Conforme este instrumento, pelo menos uma reposta "Sim" em uma destas questões, permitiu que os pesquisadores continuassem com as perguntas de "A à $\mathrm{G}^{\text {" para as }}$ 41 gestantes $(57,7 \%)$. Desta forma, conforma a Tabela 2, verifica-se que $60,9 \%$ das gestantes tiveram mudança no apetite e 53,7\% relataram ter problemas de sono.

Quanto ao risco de depressão, após ter aplicado o teste Qui-quadrado com correção de Yates, verificou-se que $52,1 \%$ das gestantes não apresentavam risco de depressão (p-valor: 0,004), porém 29,6\% apresentou risco moderado (p-valor: 0,004) (Tabela 3).

\section{DISCUSSÃO}

À luz dos resultados, verificou-se que grande parcela das gestantes se encontravam no segundo trimestre de gestação. De fato várias avaliações durante a gravidez, podem mostrar diferenças nas taxas de depressão e ansiedade, com maior prevalência destes transtornos no terceiro trimestre de gestação ${ }^{(3)}$. 
Não

(B) Teve problemas de sono quase todas as noites?

\begin{tabular}{llll} 
Sim & 22 & 53,7 \\
\hline Não & 19 & 46,3 \\
\hline $\begin{array}{lll}\text { (C) Falou ou movimentou-se mais lentamente que de costume ou } \\
\text { pelo contrário, sentiu-se mais agitado ou incapaz de ficar quieto? }\end{array}$ & \\
\hline Sim & 23 & 56,1 \\
\hline Não & 18 & 43,9
\end{tabular}

(D) Sentiu-se a maior parte do tempo cansado, sem energia, quase todas os dias?

\begin{tabular}{llll} 
Sim & 31 & 75,7 \\
\hline Não & 10 & 24,3 \\
\hline (E) Sentiu-se sem valor ou com culpa, quase todos os dias? & & \\
\hline Sim & 10 & 24,3 \\
\hline Não & 31 & 75,7 \\
\hline
\end{tabular}

(F) Teve dificuldade em tomar decisões, de se concentrar ou problemas de memória quase todos os dias?

$\begin{array}{lll}\text { Sim } & 16 & 39,1 \\ \text { Não } & 25 & 60,9\end{array}$

(G) Teve por várias vezes pensamentos ruins, como seria estar morto, ou fazer mal a si mesmo?

$\begin{array}{lll}\text { Sim } & 25 & 60,9 \\ \text { Não } & 16 & 39,1\end{array}$

Fonte: Elaborada pelos autores,

Tabela 3: Risco de depressão entre gestantes, conforme instrumento "Cartão

Babel", Olinda, PE, Brasil, 2020. (N: 71)

\begin{tabular}{lcccc}
\multicolumn{1}{c}{ Riscos } & N & $\%$ & p-valor* \\
Sem risco para depressão & 37 & 52,1 & 0,004 \\
\hline Grande risco para depressão leve (3 a 4 respostas positivas) & 06 & 08,5 & 0,003 \\
\hline Grande risco para depressão moderada (5 a 7 respostas positivas) & 21 & 29,6 & 0,004 \\
\hline Grande risco para depressão grave (8 a 9 respostas positivas) & 07 & 09,8 & 0,002
\end{tabular}

Nota: * Teste Qui-Quadrado com correção de Yates

Fonte: Elaborada pelos autores,

A análise dos riscos de ansiedade evidenciou que quase metade das gestantes, apresentavam possíveis riscos de desenvolvê-la, por meio de um valor estatístico significativo. Esta condição de risco deve ser avaliada com mais acurácia pelos profissionais de saúde visto que a ansiedade pré-natal materna tem sido associada ao aumento do medo relativo ao parto $^{(9)}$, a uma preferência por parto cesáreo ${ }^{(10)}$, a taxas mais altas de transtornos alimentares $^{(11)}$ e um risco aumentado de

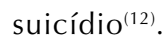

Além de complicações maternas estudos têm evidenciado possíveis implicações neonatais importantes, como nascimentos prematuros ${ }^{(13)} \mathrm{e}$ menores escores de Apgar ${ }^{(14)}$. Além disso, a ansiedade pré-natal é um risco para um baixo desenvolvimento infantil; em um estudo conduzido na
Holanda, a ansiedade pré-natal aumentou significativamente o risco de distúrbios cognitivos em crianças de 14 e 15 anos de idade ${ }^{(15)}$.

Quanto aos riscos de depressão, a análise estatística também foi bastante significativa, apesar de mais da metade das gestantes não apresentarem risco de depressão (p-valor: 0,004). Porém há de se destacar que $29,5 \%$ apresentou risco moderado (p-valor: 0,004). A prevalência global de depressão perinatal materna clinicamente significativa varia de 10 a $20 \%^{(16,17)}$; no Brasil essa taxa varia de $15 \%$ a $28 \%$ no Brasil ${ }^{(18)}$.

Está documentado que os transtornos depressivos pré-natais resultam em transtornos depressivos pós-natais, baixa capacidade de criação em crianças, bem como abortos espontâneos recorrentes elevados, crescimento pós-natal prejudicado, retardo do crescimento intrauterino, doenças diarreicas infantis frequentes, mau funcionamento social e doenças relacionadas ao sistema imunológico ${ }^{(19)}$.

Salienta-se que o período da coleta de dados ocorreu no período da pandemia da COVID-19. É importante ressaltar que a pandemia não é apenas uma crise de saúde pública, mas também social, demográfica e econômica e tem um efeito psicossocial negativo substancial em todos, incluindo mulheres grávidas ${ }^{(20)}$.

Uma vez que as mulheres tendem a relatar mais sintomas de ansiedade e depressão durante surtos de doenças do que os homens, mulheres que estão grávidas durante a pandemia de COVID-19 podem ser especialmente afetadas $^{(21)}$.

Desta forma a atenção à saúde mental na Atenção Primária é importante para antecipar a detecção de casos e interromper precocemente o processo de adoeciᄀmento; para isso, a capacitação em saúde mental dos profissionais torna-se necessária. Entretanto, apesar da alta prevalência de sofrimento mental nos pacientes aten- 
didos na rede básica de saúde, ainda não há detecção adequada. Tal fato deve-se à dificuldade apresentada pelos profissionais em diagnosticar corretamente e, por consequência, realizar o devido atendimento ${ }^{(22,23)}$

Existe então uma necessidade de pesquisas contínuas a fim de rastrear, identificar os fatores de risco e desenvolver tratamentos eficazes para gestantes com ansiedade e/ ou depressão. Nossos resultados sugerem que a detecção destes transtornos durante a gravidez pode ser valiosa para ajudar as mulheres a acessar os serviços de saúde mental necessários.

$\mathrm{Na}$ interpretação dos resultados deste estudo, é importante considerar a limitação imposta pela amostra constituída por gestantes atendidas no siste-

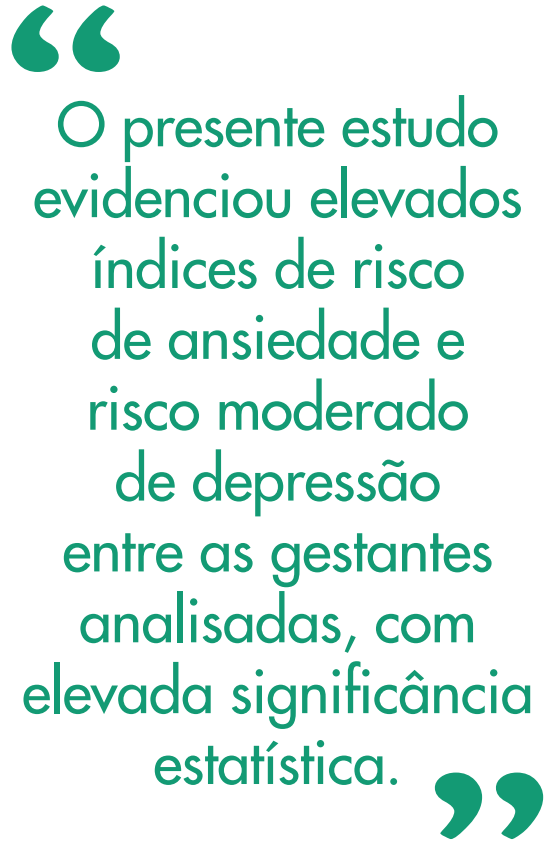

ma público de saúde e em apenas uma USF, o que impede a generalização dos resultados para o universo de mulheres grávidas.

\section{CONCLUSÃO}

O presente estudo evidenciou elevados índices de risco de ansiedade e risco moderado de depressão entre as gestantes analisadas, com elevada significância estatística. É necessário que os profissionais de saúde da Atenção Primária tenham uma visão mais ampliada acerca de transtornos de saúde mental, identificando precocemente possíveis alterações e abordando por meio da equipe multidisciplinar, possíveis estratégias de enfrentamento individual.

\section{Referências}

1. Lima MOP, Tsunechiro MA, Bonadio IC, Murata M. Sintomas depressivos na gestação e fatores associados: estudo longitudinal. Acta paul. enferm. 2017; 30(1):39-46.

2. Silva MMJ, Nogueira DA, Clapis MJ, Leite EPRC. Ansiedade na gravidez: prevalência e fatores associados. Rev. esc. enferm. USP. 2017; 51:e03253.

3. Biaggi A, Conroy S, Pawlby S, Pariante CM. Identifying the women at risk of antenatal anxiety and depression: a systematic review. J Affect Disord. 2016;191:62-77.

4. Witteveen AB, De Cock P, Huizink AC, De Jonge A, Klomp T, Westerneng M, et al. Pregnancy related anxiety and general anxious or depressed mood and the choice for birth setting: a secondary data-analysis of the DELIVER study. BMC Pregnancy Childbirth. 2016;16(1):363-76.

5. Koelewijn JM, Sluijs AM, Vrijktte TGM. Possible Relationship Between General and Pregnancy-Related Anxiety During the First Half of Pregnancy and the Birth Process: A Prospective Cohort Study. BMJ Open. 2017; 7(5):e013413. 6. Brasil. Ministério da Saúde. Atenção ao pré-natal de baixo risco / Ministério da Saúde. Secretaria de Atenção à Saúde. Departamento de Atenção Básica. Brasília: Editora do Ministério da Saúde; 2012.

7. Amorim, P. Cartão de Babel: Triagem para depressão. Rev. Bras. Psiquiatria; 2000; 22:106-15.

8. Brasil. Conselho Nacional de Saúde. Resolução CNS N 466 , de 12 de Dezembro de 2012. CNS: 2012.

9. Mahenge B, Stockl H, Likindikoki S, Kaaya S, Mbwambo J. The prevalence of mental health morbidity and its associated factors among women attending a prenatal clinic in Tanzania. Int J Gynaecol Obstet. 2015;130: 261-5.

10. Pazzagli C, Laghezza L, Capurso M, Sommella C, Lelli F, Mazzeschi C. Antecedents and consequences of fear of childbirth in nulliparous and parous women. Infant Ment Health J. 2015; 36: 62-74.

11. Dubber S, Reck C, Muller M, Gawlik S. Postpartum bonding: the role of perinatal depression, anxiety and maternal-fetal bonding during pregnancy. Arch Womens Ment Health. 2015; 18:187-95.

12. Enatescu VR, Enatescu I, Craina M, Gluhovschi A, Papava I, Romosan R, et al. State and trait anxiety as a psychopathological phenomenon correlated with postpartum depression in a Romanian sample: a pilot study. J Psychosom Obstet Gynaecol. 2014; 35: 55-61.

13. Pisoni C, Garofoli F, Tzialla C, Orcesi S, Spinillo A, Politi P, et al. Complexity of parental prenatal attachment during pregnancy at risk for preterm delivery J Matern Fetal Neonatal Med. 2016; 29: 771-6.

14. Rosenthal L, Earnshaw VA, Lewis TT, Reid AE, Lewis JB, Stasko EC, et al. Changes in experiences with discrimination across pregnanc $y$ and postpartum: age differences and consequences for mental health. Am J Public Health 2015;105: 686-93

15. Broekman BF, Chan YH, Chong YS, Kwek K, Cohen SS, Haley CL, et al. The influence of anxiety and depressive symptoms during pregnancy on birth size. Paediatr Perinat Epidemiol. 2014; 28: 116-26.

16. Korja R, Nolvi S, Kataja E.-L, Scheinin N, Junttila N, Lahtinen H, et al. The courses of maternal and paternal depressive and anxiety symptoms during the prenatal period in the FinnBrain Birth Cohort study. PLoS One. 2018; 13:e0207856. 17. Earls MF, Yogman MW, Mattson G, Rafferty J. Incorporating recognition and management of perinatal depression into pediatric practice. Pediatrics. 2019; 143:20183259HQ.

18. Moraes EV, Campos RN, Avelino MM. Depressive symptoms in pregnancy: the influence of social, psychological and obstetric aspects. Rev. Bras. Ginecol Obstet. 2016; 38(6):293-300.

19. Zegeye A, Alebel A, Gebrie A, Tesfaye B, Belay YA, Adane F, et al. Prevalence and determinants of antenatal depression among pregnant women in Ethiopia: a systematic review and meta-analysis.

BMC Pregnancy Childbirth. 2018; 18(1):462-72.

20. Kajdy A, Feduniw S, Ajdacka U, Modzelewski J, Baranowska B, Sys D, et al. Risk factors for anxiety and depression among pregnant women during the COVID-19 pandemic. Medicine. 2020; 99 (30): e21279.

21. Wang C, Pan R, Wan X, Tan Y, Xu L, Ho CS, et al. Immediate psychological responses and associated factors during the initial stage of the 2019 Coronavirus Disease (COVID-19) Epidemic among the General Population in China. Int. J. Environ. Res. Public Health. 2020;17(5): 1729-59.

22. Morais AODS, Simões VMF, Rodrigues LS, Batista RFS, Lamy ZC, Carvalho CA, et al. Sintomas depressivos e de ansiedade maternos e prejuízos na relação mãe/filho em uma coorte pré-natal: uma abordagem com modelagem de equações estruturais. Cad. Saúde Pública. 2017; 33(6): e00032016.

23. Costa DO, Souza FIS, Pedroso GC, Strufaldi MWL. Transtornos mentais na gravidez e condições do recém-nascido: estudo longitudinal com gestantes assistidas na atenção básica. Ciênc. saúde coletiva. 2018; 23(3): 691-700. 